\title{
Indicadores socioambientais na gestão integrada das sub bacias da região metropolitana de Sorocaba, SP
}

\author{
Socio-environmental indicators in the integrated river basin \\ management at the Sorocaba metropolitan region, São Paulo state, \\ southeastern Brazil
}

\author{
Fernanda Maria Soares Urquieta Leinfelder ${ }^{1}$ \\ Carina Julia Pensa Correa ${ }^{2}$ \\ Mariana Santos Leal ${ }^{3}$ \\ Kelly Cristina Tonello ${ }^{4}$
}

\begin{abstract}
Resumo
O presente estudo teve por objetivo diagnosticar a situação dos recursos hídricos das sete sub-bacias que compõe a Região Metropolitana de Sorocaba (RMS), localizada no estado de São Paulo, instituída pela Lei Complementar 1.241 de 2014, a partir da utilização de indicadores que permitiram identificar problemas socioambientais. Indicadores demográficos e de responsabilidade social, indicadores de qualidade das águas, indicadores de disponibilidade e demanda hídrica e indicadores de estado e resposta de serviços de saneamento foram obtidos para as subbacias que integram a RMS. Existem três sub-bacias que estão fortemente comprometidas com relação aos indicadores ambientais, sendo que duas dessas sub-bacias também apresentaram indicadores sociais ruins, apesar do elevado nível de riqueza. As três sub-bacias com baixo nível de riqueza, baixa taxa de urbanização e baixa densidade demográfica apresentaram resultados bons ou intermediários para os indicadores sociais, porém duas dessas sub-bacias merecem atenção devido a problemas de disponibilidade e demanda de água e poluição hídrica. Finalmente, a sub-bacia que abriga o maior manancial de abastecimento da região apresentou bons resultados para os indicadores ambientais, porém com indicadores sociais ruins. Por apresentarem características diversas, a gestão integrada das sub-bacias RMS pode ser compreendida como um grande desafio, na perspectiva de atender às diferentes necessidades das dimensões social, econômica e ambiental necessárias ao desenvolvimento. Sendo assim, esse estudo irá contribuir para identificação de linhas de atuação municipais e regionais de forma a proporcionar o desenvolvimento sustentável dessa região.

Palavras-chave: Desenvolvimento Regional. Gestão Ambiental. Recursos Hídricos.
\end{abstract}

\footnotetext{
Abstract

We aimed to diagnose the current status of water resources at the seven river basins that form the Sorocaba Metropolitan Region (SMR) in São

${ }^{1}$ Universidade Federal de São Carlos, Sorocaba, SP, Brasil. urquieta.leinfelder@gmail.com

${ }^{2}$ Universidade Federal de São Carlos, Sorocaba, SP, Brasil. carinapensa@gmail.com

${ }^{3}$ Universidade Federal de São Carlos, Sorocaba, SP, Brasil. mari_floresta@hotmail.com

${ }^{4}$ Universidade Federal de São Carlos, Sorocaba, SP, Brasil. kellytonello@yahoo.com

Artigo recebido em: 05/06/2017. Aceito para publicação em: 11/09/2019.
} 
Paulo state, southeastern Brazil, by using indicators that would enable us to identify socio-environmental issues. Indicators of demography and social responsibility, water quality, water supply and demand, as well as state and response indicators of sanitation services, were obtained for the SMR basins. Three basins are strongly compromised in terms of environmental indicators, and two of them also underperformed on social indicators, despite the high local wealth. The three basins that have low wealth, low urbanization rate and low demographic density yielded good or intermediary values for social indicators, yet two of those basins should be given special attention, as they were revealed to be having issues related not only to water supply and demand but also to water pollution. Lastly, the basin that harbors the largest water supply in the region yielded good values for environmental indicators, yet it also yielded poor results for social ones. As the SMR basins have diverse features, their integrated management may pose a huge challenge, insofar as it should meet different social, economic and environmental needs that are necessary for development. In that sense, the present study should contribute to identifying actions that should be taken at the municipal and regional scales in order to foment sustainable development in the region.

Keywords: Regional Development. Environmental Management. Water Resources.

\section{Introdução}

A Região Metropolitana de Sorocaba (RMS), localizada no estado de São Paulo, foi instituída com o objetivo de promover a integração do planejamento e da execução das funções públicas de interesse comum aos entes públicos atuantes nessa região. Sua institucionalização justifica-se pela importância nas esferas, como, a econômica, a densidade demográfica, a significativa conurbação, a agregação socioeconômica e as funções urbanas diversas e especializadas de seus municípios.

Para Masullo e Lopes (2017), o que se tornou uma questão central nos últimos estudos sobre as Regiões Metropolitanas foi a forma de tratamento e análise das ações dos agentes envolvidos no planejamento e gestão das regiões, por estes pensarem o local de forma segregada e isolada e não ampliando e flexibilizando os interesses entre os municípios que constituem tal região. De acordo com Tischer e Polette (2016), a ausência de indicadores socioambientais, econômicos e de governança no Brasil, tem sido um dos 
grandes desafios para a implementação efetiva dos instrumentos das políticas públicas e ambientais e urbanas no país.

Para avaliar a proposta de constituição da RMS, o Núcleo de Planejamento do Sudoeste Paulista desenvolveu um estudo que considerou indicadores das dimensões demográfica, econômica e social. No entanto, não foram considerados indicadores de gestão de recursos hídricos, que seriam de extrema importância para identificar os principais problemas ambientais das sub-bacias que integram essa região. Algo extremamente importante quando o assunto é sustentabilidade, uma vez que os corpos hídricos tem sofrido cada vez mais pressões por parte da sociedade, como também foi discutido por Carvalho et al. (2011).

Indicadores são muito utilizados em ações de monitoramento, uma vez que são projetados para simplificar informações e possibilitar o acompanhamento temporal das transformações ocorridas em uma região, como por exemplo, uma bacia hidrográfica (CBH-ALPA, 2013). Gomes e Malheiros (2012) e Malheiros et al. (2008), enfatizam a importância dos indicadores como ferramenta para o estabelecimento de uma visão do conjunto, e identificação de entraves relacionados a políticas públicas que envolvam aspectos de cunho econômico, social e ambiental (CARVALHO; CURI, 2016). Indicadores eficazes podem mostrar relações de causa e efeito, permitindo assim a imediata tomada de decisão para uma intervenção (NAEEM; WRIGHT, 2003).

No contexto da gestão dos recursos hídricos emerge um processo decisório complexo repleto de variáveis e dados que necessitam ser estruturados de modo que possa ser capaz de contribuir para um melhor planejamento e gerenciamento da situação hídrica no intuito de contribuir para a melhoria e definição de estratégias de gestão pública (CARVALHO; CURI, 2016).

O Sistema Integrado de Gerenciamento de Recursos Hídricos do Estado de São Paulo adotou um Banco de Indicadores para a elaboração do Relatório de Situação dos Recursos Hídricos. O relatório objetiva monitorar 
o balanço entre a quantidade e a qualidade dos recursos hídricos e avaliar a eficiência dos investimentos e do Plano Estadual de Recursos Hídricos na recuperação da qualidade e no aumento da oferta de água (SIGRH, 2013).

A utilização desses indicadores socioambientais, que visam avaliar a situação dos recursos hídricos, permite diagnosticar os aspectos demográficos e de responsabilidade social, a qualidade da água, a demanda e disponibilidade hídrica e os serviços de saneamento básico. Esses indicadores são parâmetros básicos que se aplicam à análise em todas as Unidades de Gerenciamento de Recursos Hídricos (UGRHIs), e seus resultados permitem direcionar esforços para linhas estratégicas de desenvolvimento sustentável municipal e regional. Nesse contexto, o presente trabalho teve como objetivo diagnosticar a situação dos recursos hídricos das sete sub-bacias que compõe a Região Metropolitana de Sorocaba (RMS), localizada no estado de São Paulo, a partir da utilização de indicadores que permitiram identificar problemas socioambientais.

\section{Metodologia}

O trabalho foi realizado na Região Metropolitana de Sorocaba, instituída pela Lei $\mathrm{n}^{\circ}$. 1.241/2014 e que abrange uma área de 9.821,32 km2 possui uma população de 1.805.473 habitantes e está localizada próxima à capital do estado de São Paulo (São Paulo, 2014). Essa região compreende 26 municípios e dentro de seus limites territoriais são encontradas três Unidades de Gerenciamento de Recursos Hídricos.

A UGRHI 10-Sorocaba e Médio Tietê, se localiza na área mais populosa, urbanizada e industrial da RMS, com a menor cobertura vegetal (13,57\% de seu território) e um déficit de cobertura vegetal nas áreas de preservação permanente de 77\% a 95\% (CBH-SMT, 2013; SSRH, 2011). Já a UGRHI 11-Ribeira do Iguape e Litoral Sul, está localizada em área prioritária de conservação, com alto índice de vegetação natural (66,2\% de 
sua superfície) e atividades de agropecuária, mineração e turismo (SSE, 2014).

Por fim, a UGRHI 14 - Alto Paranapanema, possui atividades de agropecuária, silvicultura, indústria extrativa, turismo, agroindústria e vegetação natural (20\% de sua superfície), onde $15 \%$ destas áreas são protegidas por legislação específica, sendo considerada, portanto uma região de conservação (CBH-PCJ, 2014; CBH-ALPA, 2013).

As três UGRHIs contemplam sete sub-bacias: Médio Tiete Médio, Baixo Sorocaba, Médio Sorocaba, Médio Tietê Superior, Alto Sorocaba, Alto Itapetininga e Juquiá, que também divergem entre si quanto as suas características. Enquanto umas são essencialmente urbanas, apresentando elevado nível de riqueza decorrente do desenvolvimento da indústria, de atividades minerárias e do setor de serviços, outras apresentam baixa taxa de urbanização e elevado percentual de cobertura vegetal, com atividades econômicas voltadas para a agricultura, pecuária, reflorestamento e mineração (Figura 1).

Figura 1. Unidades de Gerenciamento de Recursos Hídricos (UGRHIs), suas correspondentes subbacias hidrográficas e os municípios da RMS, Sorocaba, SP.
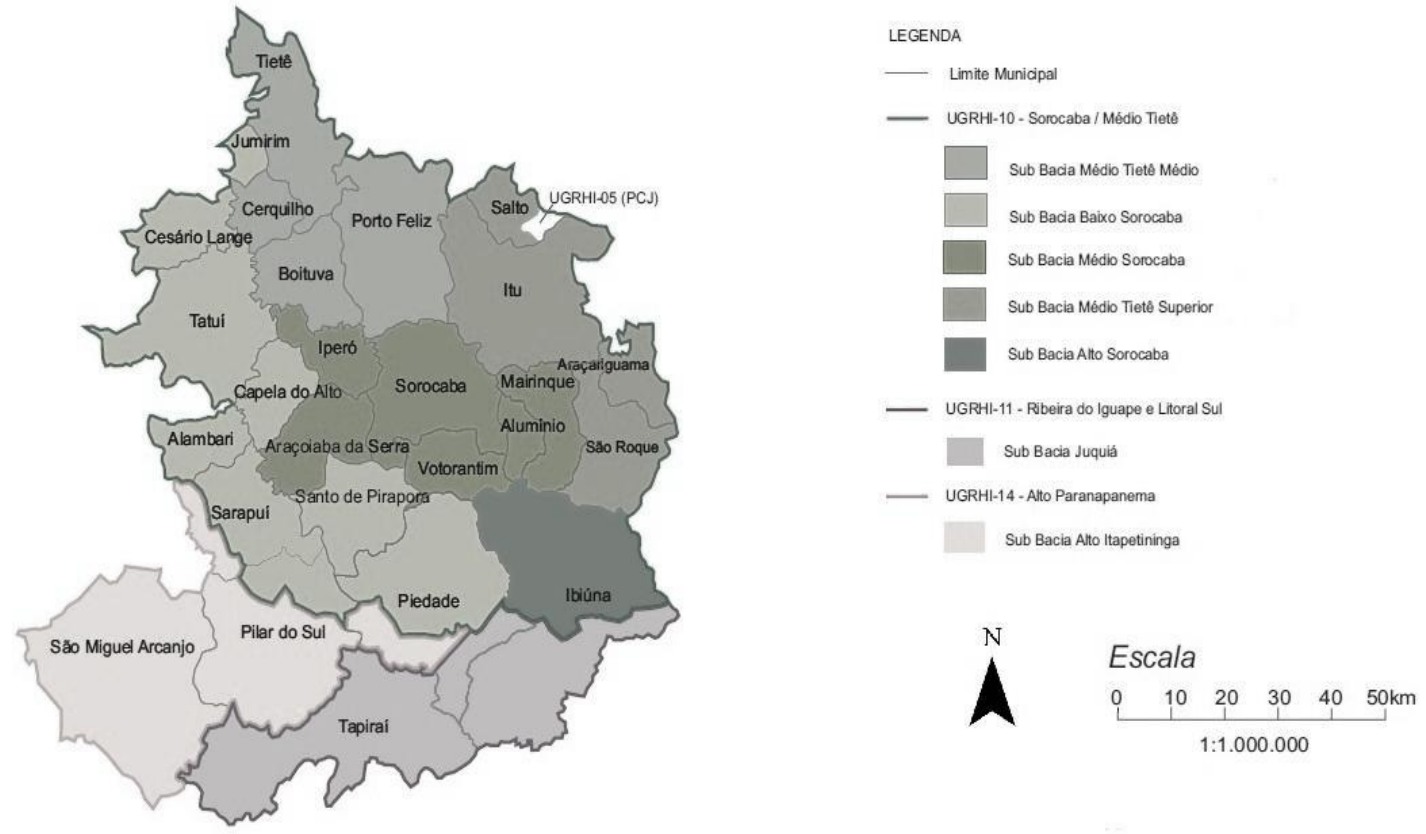

Org: do Autor, 2017. 


\section{Avaliação das sub-bacias hidrográficas}

Os indicadores selecionados tiveram que atender a dois critérios estabelecidos neste trabalho: deveriam ser aplicados à análise da situação dos recursos hídricos em todas as UGRHIs (parâmetros básicos), e apresentar valores que permitissem a classificação das sub-bacias em faixas de referência estabelecidas pelos órgãos governamentais geradores dos dados ou pelo Conselho Regional dos Recursos Hídricos do Estado de São Paulo.

Assim sendo, 23 indicadores foram selecionados e classificados a partir do Banco de Indicadores nas seguintes categorias: Força-Motriz (cinco indicadores de demografia e responsabilidade social), Estado (13 indicadores referentes à qualidade da água, disponibilidade e demanda hídrica e saneamento) e Resposta (cinco indicadores de saneamento básico). Compreender a inter-relação entre essas categorias de indicadores permite emitir respostas para reduzir as pressões diretas ou efeitos indiretos no estado do meio ambiente (SIGRH, 2013). As categorias de pressão e impacto não foram utilizadas por não apresentarem, conjuntamente, parâmetros básicos aplicáveis a todas as sub-bacias e valores que permitissem a sua classificação em faixas de referência.

Os indicadores demográficos da força motriz foram calculados utilizando a metodologia descrita no Banco de Indicadores e classificados de acordo com as faixas de referências, enquanto que para a análise do Índice Paulista de Responsabilidade Social (IPRS) foi considerada a moda dos resultados obtidos pelos municípios que as compõem (Tabela 1).

Dentro da categoria Estado (Tabela 2), para a análise dos indicadores de qualidade da água, foram considerados os resultados obtidos nos pontos de monitoramento presentes no Relatório de Qualidade das Águas Superficiais do Estado de São Paulo (CETESB, 2014a). 
Tabela 1. Indicadores demográficos e de responsabilidade social referentes à Força Motriz aplicados nas sub-bacias da Região Metropolitana de Sorocaba, SP.

\begin{tabular}{|c|c|c|}
\hline Indicador & Faixas de Referência & Critério de Avaliação \\
\hline $\begin{array}{l}\text { Taxa Geométrica de Crescimento } \\
\text { Anual (TGCA): representa o } \\
\text { crescimento médio da população } \\
\text { residente numa região em um } \\
\text { determinado período de tempo, } \\
\text { indicando o ritmo de crescimento } \\
\text { populacional (SEADE, 2014a). }\end{array}$ & $\begin{array}{c}<0 \\
\geq 0 \mathrm{e}<0,6 \\
\geq 0,6 \mathrm{e}<1,2 \\
\geq 1,2 \mathrm{e}<1,8 \\
\geq 1,8 \mathrm{e}<2,4 \\
\geq 2,4 \mathrm{e}<3 \\
\geq 3 \% \text { a.a }\end{array}$ & - \\
\hline $\begin{array}{l}\text { População Total: é a totalidade dos } \\
\text { indivíduos que residem em uma } \\
\text { determinada localidade (SEADE, } \\
\text { 2014b). }\end{array}$ & $\begin{array}{c}\leq 50.000 \\
>50.000 \mathrm{e} \leq 100.000 \\
>100.000 \mathrm{e} \leq 500.000 \\
>500.000 \mathrm{e} \leq 1.000 .000 \\
>1.000 .000\end{array}$ & - \\
\hline
\end{tabular}

Densidade Demográfica: número de habitantes residentes em uma região geográfica em determinado momento em relação à área da mesma, indicando a intensidade de ocupação de um território (SEADE, 2014c).

$$
\leq 10
$$

$>10$ e $\leq 30$

$>30$ e $\leq 50$

$>50$ e $\leq 70$

$>70$ e $\leq 100$

$>100$ e $\leq 1.000$

$>1.000 \mathrm{hab} / \mathrm{km}^{2}$

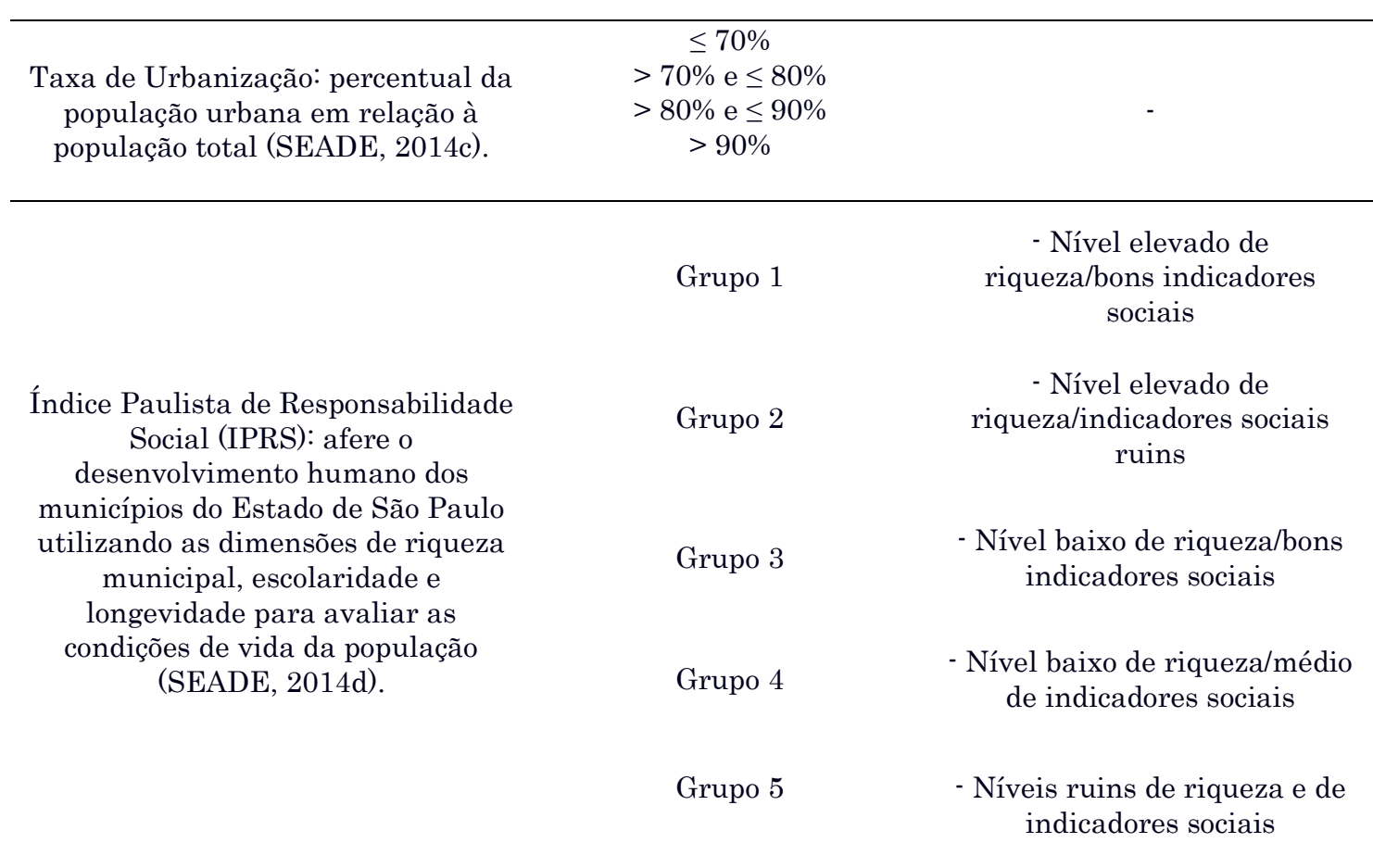

Fonte: Adaptado de SEADE (2014a), SEADE (2014b), SEADE (2014c), SEADE (2014d) 
Tabela 2. Indicadores de qualidade da água referentes ao Estado aplicados nas sub-bacias da Região Metropolitana de Sorocaba, SP.

\begin{tabular}{ccc}
\hline Indicador & $\begin{array}{c}\text { Faixas de } \\
\text { Referência }\end{array}$ & Critério de Avaliação \\
\hline Índice de Qualidade das Águas (IQA): indica & $79<\mathrm{IQA} \leq 100$ & ÓTIMO \\
a contaminação dos corpos hídricos & $51<\mathrm{IQA} \leq 79$ & BOM \\
ocasionada pelo lançamento de efluentes & $36<\mathrm{IQA} \leq 51$ & REGULAR \\
domésticos (CETESB 2014a). & $19<\mathrm{IQA} \leq 36$ & RUIM \\
\hline
\end{tabular}

Índice de Qualidade das Águas Brutas para fins de Abastecimento Público (IAP): índice composto pela ponderação do IQA e do índice de substâncias tóxicas organolépticas, que avalia as substâncias tóxicas e as variáveis que afetam a qualidade organoléptica da água, advinda, principalmente, de fontes difusas (CETESB, 2014a).

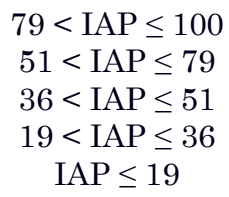

Índice de Qualidade das Águas para a Proteção da Vida Aquática (IVA): qualidade das águas para fins de proteção da fauna e flora, considerando a presença e a concentração de contaminantes tóxicos e seu efeito sobre os organismos aquáticos (CETESB, 2014a).

$\begin{array}{cc}\text { IVA } \leq 2,5 & \text { ÓTIMO } \\ 2,6<\text { IVA } \leq 3,3 & \text { BOM } \\ 3,4<\text { IVA } \leq 4,5 & \text { REGULAR } \\ 4,6<\text { IVA } \leq 6,7 & \text { RUIM } \\ \text { IVA } \geq 6,8 & \text { PÉSSIMO }\end{array}$

Índice do Estado Trófico (IET): indica o grau de enriquecimento da água por nutrientes ou grau de trofia do corpo d'água e o efeito relacionado ao crescimento excessivo de algas e infestação por macrófitas aquáticas (CETESB, 2014a).

$$
\begin{gathered}
\mathrm{IET} \leq 47 \\
47<\mathrm{IET} \leq 52 \\
52<\mathrm{IET} \leq 59 \\
59<\mathrm{IET} \leq 63 \\
63<\mathrm{IET} \leq 67 \\
\mathrm{IET}>67
\end{gathered}
$$
ULTRAOLIGOTRÓFICO OLIGOTRÓFICO MESOTRÓFICO EUTRÓFICO SUPEREUTRÓFICO HIPEREUTRÓFICO

Fonte: Adaptado de CETESB (2014a)

Para a análise da disponibilidade e demanda hídrica (Tabela 3) utilizaram-se dados de demandas hídricas outorgadas (superficial e subterrânea), a partir de consulta ao Banco de Outorgas do Departamento de Águas e Energia Elétrica do Estado de São Paulo (DAEE, 2015). Os dados das vazões de referência das sub-bacias não estavam disponíveis no Plano Estadual de Recursos Hídricos do Estado de São Paulo 2004-07, portanto o Software de Regionalização Hidrológica (SIGRH, 2015) foi a ferramenta 
encontrada para realizar o cálculo das mesmas de modo alternativo. A partir dos valores de Qmédio (vazão média de água na bacia durante o ano), Q95\% (vazão associada à permanência de 95\% no tempo) e Q7,10 (vazão mínima superficial registrada em 7 dias consecutivos em um período de retorno de 10 anos), foi possível realizar os cálculos de acordo com a metodologia descrita no Banco de Indicadores.

Tabela 3. Indicadores de disponibilidade e demanda hídrica referentes ao Estado aplicados nas sub-bacias da Região Metropolitana de Sorocaba, SP.

\begin{tabular}{|c|c|c|}
\hline $\begin{array}{l}\text { Disponibilidade per capita - Qmédio em } \\
\text { relação à população total: é potencial de } \\
\text { água, em termos de volume per capita ou de } \\
\text { reservas sociais, que permite correlacionar a } \\
\text { disponibilidade de água em relação à } \\
\text { população total, caracterizando a riqueza ou } \\
\text { pobreza de água numa determinada região } \\
\text { (DAEE, } 2015 \text { e SEADE, 2014b). }\end{array}$ & $\begin{array}{l}\text { Qmédio }>2.500 \\
2.500 \geq \text { Qmédio } \geq 1.500 \text { Qmédio }< \\
1.500\left(\mathrm{~m}^{3} / \text { hab.ano }\right)\end{array}$ & $\begin{array}{l}\text { BOA } \\
\text { ATENÇÃO } \\
\text { CRÍTICA }\end{array}$ \\
\hline $\begin{array}{l}\text { Demanda Total Superficial e Subterrânea } \\
\text { (DTSSQ95\%) em relação à disponibilidade } \\
\text { Q95\%: é o balanço entre a demanda total } \\
\text { (superficial e subterrânea) e a } \\
\text { disponibilidade Q95\% (DAEE, 2015). }\end{array}$ & $\begin{array}{l}\text { DTSSQ } 95 \%<30 \% \\
50 \% \geq \text { DTSSQ } 95 \% \geq 30 \% \\
\text { DTSSQ } 95 \%>50 \%\end{array}$ & $\begin{array}{l}\text { BOA } \\
\text { ATENÇÃO } \\
\text { CRÍTICA }\end{array}$ \\
\hline $\begin{array}{l}\text { Demanda Total Superficial e Subterrânea } \\
\text { (DTSSQmédio) em relação à disponibilidade: é } \\
\text { o balanço entre a demanda total (superficial } \\
\text { e subterrânea) e a disponibilidade Qmédio } \\
\text { (DAEE, 2015). }\end{array}$ & $\begin{array}{l}\text { DTSS }_{\text {Qmédio }}<10 \% \\
20 \% \geq \text { DTSS }_{\text {Qmédio }} \geq 10 \% \\
\text { DTSS }_{\text {Qmédio }}>20 \%\end{array}$ & $\begin{array}{l}\text { BOA } \\
\text { ATENÇÃO }\end{array}$ \\
\hline $\begin{array}{l}\text { Demanda Superficial (DSupQ7,10) em relação } \\
\text { à vazão mínima superficial } \mathrm{Q}_{7,10} \text { : é o balanço } \\
\text { entre a demanda superficial e } \quad \text { a } \\
\text { disponibilidade } \mathrm{Q}_{7,10} \text { (DAEE, 2015). }\end{array}$ & $\begin{array}{l}50 \% \geq \operatorname{DSup}_{\mathrm{Q} 7,10} \geq 30 \% \text { DSupq } 7,10> \\
50 \%\end{array}$ & $\begin{array}{l}\text { BOA } \\
\text { ATENÇÃO } \\
\text { CRÍTICA }\end{array}$ \\
\hline $\begin{array}{l}\text { Demanda Subterrânea (DSubexpl) em relação } \\
\text { às reservas explotáveis: é o balanço entre a } \\
\text { demanda subterrânea e a disponibilidade } \\
\text { hídrica subterrânea (DAEE, 2015). }\end{array}$ & $\begin{array}{l}\text { DSubexpl }_{\text {e }}<30 \% \\
50 \% \geq \text { DSubexpl } \geq 30 \% \text { DSub }_{\text {expl }}> \\
50 \%\end{array}$ & $\begin{array}{l}\text { BOA } \\
\text { ATENÇÃO } \\
\text { CRÍTICA }\end{array}$ \\
\hline
\end{tabular}

Fonte: Adaptado de DAEE (2015), SEADE (2014b) 
Em seguida, as sub-bacias hidrográficas foram classificadas nas faixas de referência do SIGRH (2013), considerando os resultados obtidos para os indicadores: disponibilidade superficial per capita - Qmédio em relação à população total, demanda total superficial e subterrânea em relação a Q ${ }_{95 \%}\left(\right.$ DTSS $_{\text {Q95\% }}$ ), demanda total superficial e subterrânea em relação ao $Q_{\text {médio }}$ (DTSSQmédio), demanda superficial em relação a $\mathrm{Q}_{7,10}$ $\left(D_{\text {SupQ7,10 }}\right)$ e demanda subterrânea em relação às reservas explotáveis (D $\left.\mathrm{D}_{\text {Subexpl}}\right)$.

Os indicadores de estado referentes ao saneamento (Tabela 4) foram obtidos por meio dos resultados presentes no Diagnóstico sobre os Serviços de Água e Esgoto de cada município (SNIS, 2012). Os indicadores de Resposta dos serviços de saneamento básico (Tabela 5) foram obtidos no Relatório de Qualidade das Águas Superficiais do Estado de São Paulo (CETESB, 2014a). Após os cálculos, as sub-bacias foram classificadas nas faixas de referência do SIGRH (2013).

Tabela 4. Indicadores de saneamento referentes ao Estado aplicados nas sub-bacias da Região Metropolitana de Sorocaba, SP.

\begin{tabular}{ccc}
\hline Indicador & Faixas de Referência & Critério de Avaliação \\
\hline $\begin{array}{c}\text { Taxa de Cobertura do Serviço de Coleta } \\
\text { de Resíduos (TCR): é o percentual } \\
\text { estimado de população total atendida } \\
\text { por coleta de resíduo sólido domiciliar } \\
\text { em relação à população total (SNIS, } \\
\text { 2012). }\end{array}$ & $90 \%>$ TCR $\geq 50 \%$ & BOM \\
\hline $\begin{array}{c}\text { Éngotos (IAE): é o percentual estimado } \\
\text { de população total atendida por coleta } \\
\text { de efluente sanitário em relação à } \\
\text { população total (SNIS, 2012). }\end{array}$ & TCR $<50 \%$ & REGULAR \\
\hline $\begin{array}{c}\text { Índice de Perdas do Sistema de } \\
\text { Distribuição de Água (IPA): é o } \\
\text { percentual estimado de perdas do } \\
\text { sistema público de abastecimento de } \\
\text { água (SNIS, 2012). }\end{array}$ & IAE $\geq 90 \%$ & ROM \\
\hline
\end{tabular}

Fonte: Adaptado de SNIS (2012) 
Tabela 5. Indicadores de saneamento básico referentes a Resposta aplicados nas sub-bacias da Região Metropolitana de Sorocaba, SP.

\begin{tabular}{|c|c|c|}
\hline Indicador & Faixas de Referência & Critério de Avaliação \\
\hline $\begin{array}{l}\text { Índice de Qualidade de Aterro de Resíduos } \\
\text { (IQR): é obtido a partir do enquadramento das } \\
\text { instalações de tratamento ou destinação final } \\
\text { de resíduos, em termos operacionais e } \\
\text { estruturais (CETESB, 2014b). }\end{array}$ & $\begin{array}{c}0<\mathrm{IQR} \leq 7,0 \\
7,0<\mathrm{IQR} \leq 10\end{array}$ & $\begin{array}{l}\text { NÃO ADEQUADO } \\
\text { ADEQUADO }\end{array}$ \\
\hline $\begin{array}{l}\text { Proporção de efluente doméstico coletado (EDC) } \\
\text { em relação ao efluente doméstico total gerado: é } \\
\text { a porcentagem do efluente doméstico coletado } \\
\text { em relação ao efluente doméstico gerado } \\
\text { (CETESB, 2014a). }\end{array}$ & $\begin{array}{c}\mathrm{EDC}<50 \% \\
90 \%>\mathrm{EDC} \geq 50 \% \\
\mathrm{EDC} \geq 90 \%\end{array}$ & $\begin{array}{c}\text { RUIM } \\
\text { REGULAR } \\
\text { BOM }\end{array}$ \\
\hline $\begin{array}{l}\text { Proporção de efluente doméstico tratado (EDT) } \\
\text { em relação ao efluente doméstico total gerado: é } \\
\text { a porcentagem do efluente doméstico tratado } \\
\text { em relação ao efluente doméstico gerado } \\
\text { (CETESB, 2014a). }\end{array}$ & $\begin{array}{c}\mathrm{EDT}<50 \% \\
90 \%>\mathrm{EDT} \geq 50 \% \\
\mathrm{EDT} \geq 90 \%\end{array}$ & $\begin{array}{l}\text { RUIM } \\
\text { REGULAR } \\
\text { BOM }\end{array}$ \\
\hline $\begin{array}{l}\text { Proporção de redução da carga orgânica } \\
\text { poluidora doméstica (RCO): é percentual de } \\
\text { remoção de carga orgânica poluidora doméstica, } \\
\text { através de tratamento, em relação à carga } \\
\text { orgânica poluidora doméstica total (CETESB, } \\
\text { 2014a). }\end{array}$ & $\begin{array}{c}\mathrm{RCO}<50 \% \\
80 \%>\mathrm{RCO} \geq 50 \% \\
\mathrm{RCO} \geq 80 \%\end{array}$ & $\begin{array}{l}\text { RUIM } \\
\text { REGULAR } \\
\text { BOM }\end{array}$ \\
\hline $\begin{array}{l}\text { Indicador de Coleta e Tratabilidade de Esgoto } \\
\text { da População Urbana de Município (ICTEM): } \\
\text { permite comparar de maneira global a eficácia } \\
\text { do sistema de esgotamento sanitário (coleta, } \\
\text { afastamento, tratamento e eficiência de } \\
\text { tratamento e a qualidade do corpo receptor dos } \\
\text { efluentes) (CETESB, 2014a). }\end{array}$ & $\begin{array}{c}0<\text { ICTEM }<2,52,6 \\
<\text { ICTEM }<5,05,1 \\
<\text { ICTEM } \leq 7,57,6< \\
\text { ICTEM } \leq 10\end{array}$ & $\begin{array}{l}\text { PÉSSIMO } \\
\text { RUIM } \\
\text { REGULAR } \\
\text { BOM }\end{array}$ \\
\hline
\end{tabular}

\section{Resultados e Discussão}

Os indicadores das sete sub-bacias foram calculados e sintetizados na

\section{Tabela 6.}


Tabela 6. Resultados dos indicadores socioambientais em cada sub-bacia: Médio Tiete Médio (MTM), Baixo Sorocaba (BS) Médio Sorocaba (MS), Médio Tietê Superior (MTS), Alto Sorocaba (AS), Alto Itapetininga (AI) e Juquiá (JUQ), na Região Metropolitana de Sorocaba, SP.

\begin{tabular}{|c|c|c|c|c|c|c|c|c|}
\hline & \multirow{2}{*}{ Indicador } & \multicolumn{7}{|c|}{ Sub-bacia } \\
\hline & & MTM & BS & MS & MTS & AS & $\mathrm{AI}$ & JUQ \\
\hline \multirow{5}{*}{ 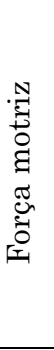 } & $\begin{array}{l}\text { TGCA } \\
(\% \text { a.a })\end{array}$ & 1,4 & 1,1 & 1,3 & 1,1 & 0,8 & 0,4 & $-0,6$ \\
\hline & $\begin{array}{l}\text { PT } \\
\text { (x 1000 hab) }\end{array}$ & 183,3 & 259,6 & 851,2 & 371,3 & 73,3 & 58,8 & 7,8 \\
\hline & $\begin{array}{l}\mathrm{DD} \\
\left(\mathrm{hab} / \mathrm{km}^{2}\right)\end{array}$ & 137,0 & 104,7 & 628,9 & 303,1 & 69,3 & 36,5 & 10,4 \\
\hline & TU (\%) & 90,7 & 78,0 & 95,0 & 95,0 & 35,0 & 73,0 & 71,5 \\
\hline & $Q_{\text {médio (m³/hab.ano) }}$ & $\underset{* * *}{1215,4}$ & $\underset{* *}{2355,8}$ & $\begin{array}{c}323,9 \\
* * *\end{array}$ & $\begin{array}{c}742,9 \\
* * *\end{array}$ & $\begin{array}{c}2506,8 \\
*\end{array}$ & $\begin{array}{c}1469,4 \\
* *\end{array}$ & $\begin{array}{c}29334,5 \\
*\end{array}$ \\
\hline \multirow{8}{*}{$\begin{array}{l}0 \\
\frac{0}{0} \\
\text { in } \\
\text { in } \\
\text { r }\end{array}$} & $\begin{array}{l}\text { DTSS }_{\text {Q95\% }} \\
\text { (\%) }\end{array}$ & $\begin{array}{l}73,7 \\
* * *\end{array}$ & $\begin{array}{c}32,8 \\
* *\end{array}$ & $\begin{array}{l}119,0 \\
* * *\end{array}$ & $\begin{array}{l}77,7 \\
* * *\end{array}$ & $\begin{array}{l}3,7 \\
*\end{array}$ & $\begin{array}{l}33 \\
* *\end{array}$ & $\begin{array}{c}0,4 \\
*\end{array}$ \\
\hline & $\begin{array}{l}\text { DTSS }_{\text {Qmédio }} \\
(\%)\end{array}$ & $\begin{array}{l}27,1 \\
* * *\end{array}$ & $\begin{array}{c}12,1 \\
* *\end{array}$ & $\begin{array}{l}43,8 \\
* * *\end{array}$ & $\begin{array}{l}28,6 \\
* * *\end{array}$ & $\underset{*}{1,4}$ & $\underset{* *}{14,1}$ & $\begin{array}{c}0,2 \\
*\end{array}$ \\
\hline & $\begin{array}{l}\operatorname{DSup}_{\mathrm{Q} 7,10} \\
(\%)\end{array}$ & $\begin{array}{l}88,6 \\
* * *\end{array}$ & $\begin{array}{l}52,0 \\
* * *\end{array}$ & $\begin{array}{c}101,9 \\
* * *\end{array}$ & $\begin{array}{c}104,3 \\
* * *\end{array}$ & $\begin{array}{c}2,3 \\
*\end{array}$ & $\begin{array}{c}41,0 \\
* *\end{array}$ & $\begin{array}{l}0,2 \\
*\end{array}$ \\
\hline & $\begin{array}{l}\text { DSub }_{\exp } \\
(\%)\end{array}$ & $\begin{array}{l}43,8 \\
* *\end{array}$ & $\begin{array}{l}7,8 \\
*\end{array}$ & $\begin{array}{c}173,5 \\
* * *\end{array}$ & $\begin{array}{c}24,8 \\
*\end{array}$ & $\begin{array}{c}2,9 \\
*\end{array}$ & $\begin{array}{c}14,6 \\
*\end{array}$ & $\begin{array}{c}0,3 \\
*\end{array}$ \\
\hline & IAA (\%) & $\begin{array}{c}87,5 \\
* *\end{array}$ & $\begin{array}{c}86,2 \\
* *\end{array}$ & $\begin{array}{l}97,0 \\
*\end{array}$ & $\begin{array}{c}85,8 \\
* *\end{array}$ & $\begin{array}{l}39,9 \\
* * *\end{array}$ & $\begin{array}{c}74,6 \\
* *\end{array}$ & $\begin{array}{c}64,7 \\
* *\end{array}$ \\
\hline & TCR (\%) & $\begin{array}{c}97,4 \\
*\end{array}$ & $\begin{array}{c}92,3 \\
*\end{array}$ & $\begin{array}{c}29,9 \\
* * *\end{array}$ & $\begin{array}{c}100,0 \\
*\end{array}$ & $\begin{array}{c}98,2 \\
*\end{array}$ & $\begin{array}{c}100,0 \\
*\end{array}$ & $\begin{array}{c}90,2 \\
*\end{array}$ \\
\hline & IAE (\%) & $\begin{array}{c}82,6 \\
* *\end{array}$ & $\begin{array}{c}68,2 \\
* *\end{array}$ & $\begin{array}{l}92,5 \\
*\end{array}$ & $\begin{array}{l}79,4 \\
* *\end{array}$ & $\begin{array}{c}19,4 \\
* * *\end{array}$ & $\begin{array}{l}57,9 \\
* *\end{array}$ & $\begin{array}{l}57,0 \\
* *\end{array}$ \\
\hline & IPA (\%) & $\begin{array}{c}37,9 \\
* * \\
\end{array}$ & $\begin{array}{l}48,3 \\
* * * \\
\end{array}$ & $\begin{array}{c}35,4 \\
* * \\
\end{array}$ & $\begin{array}{c}46,0 \\
* * *\end{array}$ & $\begin{array}{c}51,1 \\
* * *\end{array}$ & $\begin{array}{c}37,3 \\
* * \\
\end{array}$ & $\begin{array}{c}39,4 \\
* * \\
\end{array}$ \\
\hline \multirow{5}{*}{$\begin{array}{l}\frac{\pi}{10} \\
\text { in } \\
0 \\
0 \\
0 \\
0 \\
0 \\
02\end{array}$} & IQR & $\begin{array}{c}9,8 \\
*\end{array}$ & $\begin{array}{c}9,7 \\
*\end{array}$ & $\begin{array}{c}8,7 \\
*\end{array}$ & $\begin{array}{c}8,8 \\
*\end{array}$ & $\begin{array}{c}8,0 \\
*\end{array}$ & $8,5^{*}$ & $\begin{array}{c}9,0 \\
*\end{array}$ \\
\hline & EDC (\%) & $\begin{array}{c}95,3 \\
*\end{array}$ & $\begin{array}{c}78,3 \\
* *\end{array}$ & $\begin{array}{c}71,3 \\
* *\end{array}$ & $\begin{array}{c}77,8 \\
* *\end{array}$ & $\begin{array}{c}55,0 \\
* *\end{array}$ & $\begin{array}{c}75,5 \\
* *\end{array}$ & $\begin{array}{c}73,0 \\
* *\end{array}$ \\
\hline & EDT (\%) & $\begin{array}{l}39,6 \\
* * *\end{array}$ & $\begin{array}{l}36,6 \\
* * *\end{array}$ & $\begin{array}{c}25,4 \\
* * *\end{array}$ & $\begin{array}{c}65,5 \\
* *\end{array}$ & $\begin{array}{c}50,5 \\
* *\end{array}$ & $\begin{array}{l}34,6 \\
* * *\end{array}$ & $\begin{array}{c}56,1 \\
* *\end{array}$ \\
\hline & RCO (\%) & $\begin{array}{c}60,1 \\
* *\end{array}$ & $\begin{array}{c}57,5 \\
* *\end{array}$ & $\begin{array}{l}38,8 \\
* * *\end{array}$ & $\begin{array}{c}23,3 \\
* * *\end{array}$ & $\begin{array}{l}49,5 \\
* * *\end{array}$ & $\begin{array}{c}65,4 \\
* *\end{array}$ & $\begin{array}{l}43,9 \\
* * *\end{array}$ \\
\hline & ICTEM & $\begin{array}{l}7,3 \\
* *\end{array}$ & $\begin{array}{l}7,0 \\
* *\end{array}$ & $\begin{array}{l}5,0 \\
* *\end{array}$ & $\begin{array}{l}3,5 \\
* *\end{array}$ & $\begin{array}{l}6,0 \\
* *\end{array}$ & $\begin{array}{l}7,2 \\
* *\end{array}$ & $\begin{array}{l}5,9 \\
* *\end{array}$ \\
\hline
\end{tabular}

* avaliação: *** ruim/crítica, ** atenção/regular, * bom.

\section{Médio Tietê Médio}

A sub-bacia apresenta elevada concentração e crescimento populacional, com características essencialmente urbanas. Nessa região predominaram municípios com baixo nível de riqueza, mas com bons indicadores sociais. A expansão dos principais eixos rodoviários da RMS 
exerce pressões para o crescimento populacional, proporcionando o aumento da dimensão riqueza quando comparado com resultados dos anos de 2008 e 2010. A disponibilidade superficial de água per capita é 30,6\% menor do que a requerida por habitante. A demanda total de água é crítica, principalmente com relação à demanda de águas superficiais, que, em períodos de estiagem, pode alcançar até 97,3\% da vazão mínima de referência. A demanda de água subterrânea também requer estado de atenção, com o consumo de $43,8 \%$ das reservas totais explotáveis. No trecho do rio Tietê dentro dessa sub-bacia, a qualidade da água apresenta IQA ruim, IVA péssimo e IET hipereutrófico devido ao baixo percentual de tratamento do efluente doméstico gerado (39,6\%). Assim o IQA encontrado foi ruim, o IVA péssimo e o IET hipereutrófico. Já o rio Sorocaba obteve IQA bom devido ao processo natural de autodepuração da água, no entanto apresentou IAP ruim, IAV regular e IET oligotrófico. Esta sub-bacia recebe ainda os esgotos não tratados das sub-bacias do Médio Tietê Superior e do Médio Sorocaba.

\section{Baixo Sorocaba}

$\mathrm{Na}$ sub-bacia do Baixo Sorocaba a disponibilidade superficial de água per capita é $34,6 \%$ maior do que a requerida por habitante no período de um ano. A demanda total de água requer estado de atenção, principalmente com relação à demanda de águas superficiais que é crítica em períodos de estiagem e pode alcançar 51,8\% da vazão mínima de referência, com potenciais prejuízos para os sistemas aquáticos e uso múltiplo das águas. A demanda de água subterrânea também requer estado de atenção nessa subbacia, com o consumo de $42,4 \%$ das reservas totais explotáveis. Há corpos hídricos poluídos devido ao baixo percentual de tratamento do efluente doméstico gerado (36,6\%), como o rio Pirapora que apresentou IQA regular, IAP regular, IAV regular e IET mesotrófico, o que compromete o 
abastecimento de água em vários municípios. No entanto o rio Sarapuí, apresentou IQA bom, IAP regular, IAV ótimo e IET ultraoligotrófico.

\section{Médio Sorocaba}

Observa-se uma predominância de municípios com elevado nível de riqueza nas sub-bacias Médio Sorocaba, assim como no Médio Tietê Superior, e no Alto Sorocaba. No entanto, essas regiões não apresentaram bons indicadores sociais, onde o desenvolvimento econômico não significou distribuição de renda e melhorias sociais. Apenas os municípios de Sorocaba, Itu e Salto apresentaram elevado nível de riqueza e bons indicadores sociais, com alta longevidade da população. Já município de Araçoiaba da Serra, apesar de não apresentar elevado nível de riqueza, exibiu bons indicadores sociais. Quando observada a disponibilidade superficial de água per capita, nota-se que ela é $81,5 \%$ menor do que a mínima requerida por habitante no período de um ano. Em períodos de estiagem, a demanda superficial de água desta sub-bacia pode ultrapassar em $88,3 \%$ a vazão mínima de referência. Como consequência, foram concedidas outorgas para uso dos recursos hídricos que ultrapassaram em 73,6\% o total de reservas explotáveis. A escassez qualitativa é outro problema crítico, pois, além da demanda total de água ser elevada, essa sub-bacia apresentou IQA regular, IVA ruim e pontos com o IET eutrofizado e supereutrofizado ao longo do rio Sorocaba. A qualidade mostrou-se regular com sensível deterioração principalmente no trecho que atravessa os municípios de Sorocaba e Votorantim, decorrente do baixo percentual de tratamento do efluente doméstico total gerado $(25,4 \%)$ e do recebimento das cargas poluidoras do rio Pirajibu. A baixa taxa de cobertura do serviço de coleta de resíduos (29,9\%) é outro fator agravante para a condição de saneamento. Ressalta-se que ela contém o maior contingente populacional da RMS (aproximadamente 50\%) e, portanto, 7,5\% de esgoto não coletado nesta sub-bacia representam o não atendimento a 
uma população de 63.840 habitantes. Sua demanda de água é em grande parte atendida pela água produzida na sub-bacia do Alto Sorocaba.

\section{Médio Tietê Superior}

A disponibilidade superficial per capita de água na sub-bacia do Médio Tietê Superior é 57,5\% menor do que a mínima requerida por habitante no período de um ano. A demanda total de água é crítica, principalmente a demanda superficial. Em períodos de estiagem, ela pode ultrapassar em 18,3\% da vazão mínima de referência, o que acarreta situações de escassez hídrica em municípios dessa sub-bacia. No trecho do rio Tietê a qualidade da água foi regular apresentando IQA regular, IVA ruim e IET eutrófico. A qualidade regular da água se dá devido ao baixo percentual de remoção da matéria orgânica poluidora do efluente doméstico tratado $(23,3 \%)$ e das cargas poluidoras recebidas da Região Metropolitana de São Paulo (RMSP) e do rio Jundiaí.

\section{Alto Sorocaba}

A sub-bacia do Alto Sorocaba, bem como a do Alto Itapetininga e Juquiá, abriga áreas de preservação e mananciais de abastecimento, e possuem menores populações e adensamentos populacionais. Na sub-bacia do Alto Sorocaba a qualidade da água do rio Sorocabuçu e do rio Sorocamirim, formadores do Reservatório de Itupararanga, apresentaram bons resultados para o IQA e o IVA, no entanto o IAP foi considerado ruim e regular, respectivamente. No Reservatório de Itupararanga a qualidade encontrada foi ótima (inclusive para fins de abastecimento público), sendo que o ponto de atenção nesta sub-bacia é a poluição do rio Una, que recebe os efluentes de frigoríficos, produção de charque, fabricação de rações para animais e atividades minerárias (CETESB, 2015). A disponibilidade superficial de água per capita na sub-bacia é 43,2\% maior que a requerida 
por habitante no período de um ano. A demanda total de água nessa subbacia alcança pequenos percentuais quando comparada às vazões de referência. A demanda superficial de água pode alcançar, em período de estiagem, apenas 4,4\% da vazão mínima de referência. Os indicadores de saneamento são ruins, porém, como o seu grau de urbanização é baixo, a população que dispõe de sistemas alternativos de saneamento não é considerada nesse indicador, o qual considera apenas a população urbana atendida pelas companhias de saneamento. Porém, o baixo nível de coleta (55,0\%) e de tratamento do esgoto urbano gerado (50,5\%) é preocupante, uma vez que essa sub-bacia abrange áreas de mananciais e de preservação que são importantíssimas para o abastecimento de água da sub-bacia do Médio Sorocaba.

\section{Alto Itapetininga}

$\mathrm{Na}$ sub-bacia do Alto Itapetininga, a disponibilidade superficial de água per capita é $16,0 \%$ menor que a requerida por habitante no período de um ano. A demanda total de água requer estado de atenção, principalmente com relação à demanda de águas superficiais que em períodos de estiagem pode alcançar 40,1\% da vazão mínima de referência, com potenciais prejuízos para os sistemas aquáticos e uso múltiplo das águas. Essa subbacia apresentou IQA bom e IET eutrófico, todavia o baixo percentual de tratamento do efluente doméstico total gerado $(34,6 \%)$ é preocupante, pois essa sub-bacia é considerada uma região de conservação de áreas naturais.

\section{Juquiá}

A sub-bacia do Juquiá é uma das mais desfavorecidas da RMS, tanto em riqueza quanto aos indicadores sociais. Sua disponibilidade superficial de água per capita é $1.676,3 \%$ maior do que a requerida por habitante no 
período de um ano. A demanda total de água nessa sub-bacia alcança percentuais ínfimos quando comparada às vazões de referência. A demanda superficial de água pode alcançar, em período de estiagem, apenas $0,50 \%$ da vazão mínima de referência. Apesar do escopo deste trabalho considerar, para essa sub-bacia, apenas os índices de saneamento do município de Tapiraí, a baixa proporção de redução da carga orgânica poluidora doméstica $(43,9 \%)$ apresentada por esse município é preocupante, pois essa sub-bacia abrange áreas protegidas que desempenham importante função na preservação de ecossistemas e manutenção da elevada disponibilidade hídrica. A sub-bacia do Juquiá não pôde ser analisada quanto aos indicadores de IQA, IAP, IVA e IET, pois não havia ponto de monitoramento em seu território dentro da RMS.

\section{Considerações Finais}

As sub-bacias da RMS apresentaram grande diversidade quando considerados os resultados dos indicadores demográficos e de responsabilidade social, indicadores de qualidade das águas, indicadores de disponibilidade e demanda hídrica, indicadores de estado de saneamento e indicadores de resposta dos serviços de saneamento.

As sub-bacias do Médio Sorocaba e Médio Tietê Superior apresentaram resultados ruins para os indicadores ambientais e para os indicadores sociais, sendo essas as sub-bacias mais urbanizadas, adensadas e com o maior nível de riqueza, quando comparado às demais sub-bacias que integram a Região Metropolitana de Sorocaba.

A sub-bacia do Médio Tietê Médio também apresentou resultados ruins para os indicadores ambientais, porém com bons indicadores sociais. Essa sub-bacia apresentou a maior taxa geométrica de crescimento anual da população e possui características demográficas semelhantes às das sub- 
bacias do Médio Sorocaba e do Médio Tietê Superior, com crescente nível de riqueza, porém com retração dos indicadores sociais.

Para as sub-bacias do Médio Sorocaba, Médio Tietê Superior e Médio Tietê Médio são necessárias ações imediatas para a racionalização do uso da água, com impedimentos ou restrições à concessão de novas outorgas para o uso de águas superficiais e subterrâneas, assim como são impreteríveis respostas iminentes para melhorar o estado e as condições de saneamento básico.

As sub-bacias do Baixo Sorocaba, Alto Itapetininga e Juquiá apresentaram baixo nível de riqueza, baixa densidade demográfica e baixa taxa de urbanização em relação às demais. $O$ aumento da taxa de urbanização e da densidade populacional, sem que os indicadores socioambientais sejam geridos de forma adequada, é sentido, por exemplo, nas sub-bacias do Baixo Sorocaba e do Alto Itapetininga, que apresentaram problemas de disponibilidade e demanda hídrica e comprometimento da qualidade da água no rio Pirapora. A preocupação com essas sub-bacias é que o crescimento econômico seja acompanhado de bons indicadores sociais e ambientais.

Por apresentarem características diversas, a gestão integrada das sub-bacias da RMS pode ser compreendida como um grande desafio, na perspectiva de atender às diferentes necessidades sociais, econômicas e ambientais, para que o desenvolvimento da Região ocorra de forma mais justa e equilibrada e com o equacionamento de problemas urbanos decorrentes da falta de planejamento e concentração populacional.

\section{Referências}

CARVAlHO, J. R. M. de; CURI, W. F.; CARVALHO, E. K. M. A.; CURI, R. C. Indicadores de Sustentabilidade Hidroambiental: Um Estudo na Região do Alto Curso do Rio Paraíba, PB. Revista Sociedade \& Natureza, Uberlândia, n. 2, p. 295-310, 2011. https://doi.org/10.1590/S1982-45132011000200012 
CARVALHO, J. R. M.; CURI, W. F. Sistema de indicadores para a gestão de recursos hídricos em municípios: uma abordagem através dos métodos multicritério e multidecisor. Revista Brasileira de Gestão e Desenvolvimento Regional, v. 12, n. 2, p. 374-398, 2016.

CBH-ALPA. COMITÊ DA BACIA HIDROGRÁFICA DO ALTO PARANAPANEMA. Relatório de Situação dos Recursos Hídricos das Bacias Hidrográficas do Estado de São Paulo: Bacia Hidrográfica do Alto Paranapanema - UGRHI 14. 2013. Disponível em: <http://www.sigrh.sp.gov.br/public/uploads/documents/7458/rs-alpa-2013-vrfinal-19-12- 2013.pdf $>$. Acesso em: 24 jul 2016.

CBH-SMT. COMITE DA BACIA HIDROGRÁFICA DO ALTO PARANAPANEMA. Relatório de Situação dos Recursos Hídricos das Bacias Hidrográficas do Estado de São Paulo: Bacia Hidrográfica do Sorocaba - Médio Tietê - UGRHI 10. 2013. Disponível em: <http://www.sigrh.sp.gov.br/public/uploads/deliberation/\%5C6205/rs-06-0113.pdf>. Acesso em: 24 jul 2016.

CETESB. COMPANHIA AMBIENTAL DO ESTADO DE SÃO PAULO. Qualidade das Águas Superficiais do Estado de São Paulo - Série Relatórios 2013. São Paulo: CETESB, 2014a.

CETESB. COMPANHIA AMBIENTAL DO ESTADO DE SÃO PAULO. Inventário Estadual de Resíduos Sólidos Urbanos - Série Relatórios 2013. São Paulo: CETESB, $2014 \mathrm{~b}$.

CETESB. COMPANHIA AMBIENTAL DO ESTADO DE SÃO PAULO. Consulta ao andamento do processo de licenciamento ambiental. Disponível em: $<$ http://licenciamento.cetesb.sp.gov.br/cetesb/processo_resultado.asp $>$. Acesso em 20 ago 2016.

DAEE. DEPARTAMENTO DE ÁGUAS E ENERGIA ELÉTRICA DO ESTADO DE SÃO PAULO. Pesquisa de Usos dos Recursos Hídricos por Município. 2015. Disponível em: <http://www.aplicacoes.daee.sp.gov.br/usosrec/DaeewebexcelDpo.html>.

GOMES, P. R.; MALHEIROS, T. F. Proposta de análise de indicadores ambientais para apoio na discussão da sustentabilidade. Revista Brasileira de Gestão e Desenvolvimento Regional, v. 8, n. 2, p. 151-169, mai-ago/2012.

MALHEIROS, T. F.; PHILIPPI JR., A.; COUTINHO, S. M. V. (2008). Agenda 21 nacional e indicadores de desenvolvimento sustentável: contexto brasileiro. Revista Saúde e Sociedade, São Paulo, v. 17, n. 1, p.7-20, mar. https://doi.org/10.1590/S010412902008000100002

MASULLO, Y. A. G.; LOPES, J. A. V. Indicadores Econômicos da Região Metropolitana da Grande São Luís. Geografa, Ensino \& Pesquisa, Vol. 21 (2017), n.1, p. 30-40. https://doi.org/10.5902/2236499421050

NAEEM, S.; WRIGHT, J. P. Disentangling biodiversity effects on ecosystem functioning: deriving solutions to a seemingly insurmountable problem. Ecology Letters. v. 6, p. 567579. Abr. 2003. https://doi.org/10.1046/j.1461-0248.2003.00471.x

SÃO PAULO. Lei Complementar $N^{\circ} 1.241$ de 08 de Maio de 2014: Cria a Região Metropolitana de Sorocaba e dá providências correlatas. Disponível em: $<$ http://www.al.sp.gov.br/repositorio/legislacao/lei.complementar/2014/lei.complementar1241-08.05.2014.html>. Acesso em 4 de jul de 2016.

SEADE. FUNDAÇÃO SISTEMA ESTADUAL DE ANÁLISE DE DADOS. Informações dos Municípios Paulistas. 2014a. Consulta por localidades. Indicadores de Território e População. Disponível em: <http://www.imp.seade.gov.br/frontend/>. Acesso em: 01 jul 2016.

SEADE. FUNDAÇÃO SISTEMA ESTADUAL DE ANÁLISE DE DADOS. Informações dos Municípios Paulistas. Indicadores de População e Estatísticas Vitais. 2014b. Disponível em: <http://www.imp.seade.gov.br/frontend/>. Acesso em Jul 01, 2016.

SEADE. FUNDAÇÃO SISTEMA ESTADUAL DE ANÁLISE DE DADOS. Informações dos Municípios Paulistas. Indicadores de Caracterização do Território. 2014c. Disponível em: $<$ http://www.imp.seade.gov.br/frontend/>. Acesso em 21 jul 2016. 
SEADE. FUNDAÇÃO SISTEMA ESTADUAL DE ANÁLISE DE DADOS. Informações dos Municípios Paulistas. Indicadores de Condições de Vida. 2014d. Disponível em: $<$ http://www.imp.seade.gov.br/frontend/>. Acesso em: 01 jul 2016.

SIGRH. SISTEMA INTEGRADO DE GERENCIAMENTO DE RECURSOS HÍDRICOS DO ESTADO DE SÃO PAULO. Caderno de Indicadores para Gestão dos Recursos Hídricos do Estado de São Paulo. São Paulo: SIGRH, 2013.

SIGRH. SISTEMA INTEGRADO DE GERENCIAMENTO DE RECURSOS HÍDRICOS DO ESTADO DE SÃO PAULO. 2015. Software de Regionalização Hidrológica do Estado de São Paulo. Disponível em: <http://143.107.108.83/cgi-bin/regnet.exe?lig=podfp $>$. Acesso em: 24 jul 2016.

SNIS. SISTEMA NACIONAL DE INFORMAÇÕES SOBRE SANEAMENTO. Diagnóstico dos Serviços de Água e Esgoto - Tabela de Informações e Indicadores 2010. Brasília: MinCid/SNIS, 2012.

SSE. SECRETARIA DE SANEAMENTO E ENERGIA DO ESTADO DE SÃO PAULO. Plano Regional Integrado de Saneamento Básico para a UGRHI 11. 2011. Disponível em: <http://www.saneamento.sp.gov.br/PMS/UGRHI11/PRS_UGRHI11.pdf > Accesso: 24 Jul 2016.

SSRH - SECRETARIA DE SANEAMENTO E RECURSOS HÍDRICOS DO ESTADO DE SÃO PAULO. Plano Regional Integrado de Saneamento Básico para a UGRHI 10. São Paulo: SSRH, 2011.

TISCHER, V.; POLETTE, M. Proposta metodológica de estabelecimento de indicadores socioambientais para a zona costeira brasileira. Revista Brasileira de Gestão e Desenvolvimento Regional, v. 12, n. 2, p. 355-373. 2016. 